\title{
Impacto terapêutico da fotobiomodulação no tratamento da doença de Alzheimer:
}

\section{Uma revisão integrativa}

Therapeutic impact of photobiomodulation in the treatment of Alzheimer's disease: An integrative review

Impacto terapéutico de la fotobiomodulación en el tratamiento de la enfermedad de Alzheimer: Una revisión integradora

Recebido: 10/06/2021 | Revisado: 19/06/2021 | Aceito: 30/06/2021 | Publicado: 13/07/2021

\author{
Jane Maria Silva Carvalho \\ ORCID: https://orcid.org/0000-0003-0285-6833 \\ Universidade Brasil, Brasil \\ E-mail: jannemcarvalho@ hotmail.com \\ Laurita dos Santos \\ ORCID: https://orcid.org/0000-0002-6363-6837 \\ Universidade Brasil, Brasil \\ E-mail: laurita.santos@universidadebrasil.edu.br \\ Ricardo Scarparo Navarro \\ ORCID: https://orcid.org/0000-0003-4351-8048 \\ Universidade Brasil, Brasil \\ E-mail: ricardo.navarro@universidadebrasil.edu.br
}

\begin{abstract}
Resumo
A Doença de Alzheimer (DA) é entendida atualmente como uma série de fases, a qual inicia com um processo anormal de acúmulo de proteína no cérebro, passando pelo conhecido Transtorno Neuro Cognitivo Menor Tipo Alzheimer até os estágios perceptíveis da doença. A DA é considerado um grave problema na saúde mundial, com elevado aumento da incidência. Apesar disso, não existe um tratamento eficaz para sua cura. Porém, tratamentos não farmacológicos têm apresentado bons resultados para amenizar as perdas cognitivas e motoras apresentadas por seus pacientes, mais recentemente tem ganho destaque nos estudos, a terapia por fotobiomodulação. Deste modo, objetivo do presente estudo é realizar revisão integrativa da literatura do impacto terapêutico da fotobiomodulação no tratamento da Doença de Alzheimer. A partir da estratégia PICOS, realizou a estratégia de busca $\mathrm{cm}$ os descritores: Photobiomodulation, Alzheimer's Disease, Transcranial Photobiomodulation, Cognition, em inglês, português e espanhol, nas bases de dados PubMed, MEDLINE, LILACS e Scielo, entre janeiro de 2011 a janeiro de 2021, seguindo critérios de inclusão e exclusão dos artigos. No total, 7 artigos corresponderam a todos os critérios e objetivo do presente estudo. As pesquisas que envolvem a fotobiomodulação em pacientes com Doença de Alzheimer são ainda escassas e relativamente recentes, porém, os resultados já obtidos são significativos e promissores, pois apresentam indícios que é possível reverter alguns quadros de perdas cognitivas através de alterações celulares e melhorias circulatórias, medidas através de testes cognitivos e exames de imagem, respectivamente. O presente estudo pode concluir que a fotobiomodulação, embora ainda apresente algumas divergências quanto a sua aplicabilidade, tem um impacto positivo no tratamento da Doença de Alzheimer, pois através de inúmeras alterações que promove no cérebro, possibilita a melhora das funções cognitivas, habilidades funcionais diárias e qualidade de vida. Deste modo, mais estudos são necessários para elucidar as lacunas ainda existentes sobre a fotobiomodulação em humanos com Doença de Alzheimer.
\end{abstract}

Palavras-chave: Doença de Alzheimer; Fotobiomodulação; Laser de baixa potência.

\begin{abstract}
Alzheimer's disease (AD) is understood today as a series of stages, beginning with an abnormal process of protein accumulation in the brain, through the well-known Alzheimer's-like Minor Neuro Cognitive Disorder to the noticeable stages of the disease. AD is considered to be a serious health problem worldwide, with a high increase in incidence. Despite this, there is no effective treatment for its cure. However, non-pharmacological treatments have shown good results to mitigate the cognitive and motor losses presented by their patients, most recently has gained prominence in studies, the photobiomodulation therapy. Thus, the aim of this study is to carry out an integrative literature review of the therapeutic impact of photobiomodulation in the treatment of Alzheimer's Disease. From the PICOS strategy, the search strategy was performed using the descriptors: Photobiomodulation, Alzheimer's Disease, Transcranial Photobiomodulation, Cognition, in english, portuguese and spanish, in the PubMed, MEDLINE, LILACS and Scielo databases, between January 2011 and January 2021, following the inclusion and exclusion criteria of the articles. In
\end{abstract}


total, 7 articles met all the criteria and objective of the present study. The research involving photobiomodulation in patients with Alzheimer's disease is still scarce and relatively recent, however, the results already obtained are significant and promising, since they present indications that it is possible to reverse some pictures of cognitive losses through cellular changes and circulatory improvements, measured through cognitive tests and imaging exams, respectively. The present study can conclude that photobiomodulation, although it still presents some divergences as to its applicability, has a positive impact on the treatment of Alzheimer's disease, for through the numerous alterations it promotes in the brain, it enables the improvement of cognitive functions, daily functional abilities, and quality of life. Thus, more studies are necessary to elucidate the gaps still existing about photobiomodulation in humans with Alzheimer's disease.

Keywords: Alzheimer's disease; Photobiomodulation; Low level laser therapy.

\section{Resumen}

La Enfermedad de Alzheimer (EA) se entiende hoy como una serie de fases, que comienza con un proceso anormal de acumulación de proteínas en el cerebro, pasando por el conocido Trastorno Neuro Cognitivo Menor Tipo Alzheimer hasta las etapas notorias de la enfermedad. La EA se considera un problema grave en la salud mundial, con un elevado aumento de incidencia. A pesar de esto, no existe un tratamiento eficaz para su curación. Sin embargo, los tratamientos no farmacológicos han mostrado buenos resultados para paliar las pérdidas cognitivas y motoras que presentan sus pacientes, más recientemente, la terapia de fotobiomodulación ha ganado protagonismo en los estudios.

Así, el objetivo de este estudio es realizar una revisión bibliográfica integradora del impacto terapéutico de la fotobiomodulación en el tratamiento de la Enfermedad de Alzheimer. A partir de la estrategia PICOS, la estrategia de búsqueda se realizó mediante los descriptores: Fotobiomodulación, Enfermedad de Alzheimer, Fotobiomodulación transcraneal, Cognición, en inglés, portugués y español, en las bases de datos PubMed, MEDLINE, LILACS y Scielo, entre enero de 2011 y enero de 2021, tras la inclusión y criterios de exclusión de los artículos. En total, 7 artículos cumplieron con todos los criterios y objetivos del presente estudio. Las investigaciones que involucran fotobiomodulación en pacientes con enfermedad de Alzheimer son aún escasas y relativamente recientes, sin embargo, los resultados ya obtenidos son significativos y prometedores, ya que presentan evidencia de que es posible revertir algunos casos de pérdida cognitiva a través de alteraciones celulares y mejoras circulatorias, medidas a través de pruebas cognitivas y exámenes por imágenes, respectivamente. El presente estudio puede concluir que la FBM, aunque todavía presenta algunas divergencias en cuanto a su aplicabilidad, tiene un impacto positivo en el tratamiento de la Enfermedad de Alzheimer, ya que a través de numerosos cambios que promueve en el cerebro, permite la mejora de las funciones cognitivas, funcionales cotidianas. habilidades y calidad de vida. Por lo tanto, se necesitan más estudios para dilucidar las brechas que aún existen sobre la fotobiomodulación en humanos con la enfermedad de Alzheimer.

Palabras clave: Enfermedad de Alzheimer; Fotobiomodulación; Láser de baja potencia.

\section{Introdução}

A Doença de Alzheimer (DA) é entendida atualmente como uma série de fases, a qual inicia com um processo anormal de acúmulo de proteína no cérebro, passando pelo conhecido Transtorno Neuro Cognitivo Menor Tipo Alzheimer (Transtorno Cognitivo Leve Amnéstico) até os estágios perceptíveis da doença (inicial, intermediário e avançado) (Martinelli, 2020).

O declínio da função cognitiva é a principal característica da DA, a qual engloba diversas funções, como: funções executivas, memória, linguagem, atenção, concentração, planejamento e percepção, tornando o indivíduo incapaz de realizar suas atividades básicas e funcionais (ABRAz, 2021). Este declínio é decorrente de várias modificações neuropatológicas, como atrofia do córtex cerebral, principalmente da região fronto-temporal, e do hipocampo (Cavalcanti; Engelhardt, 2012).

Em pacientes com DA são observados dois tipos de alterações nos mecanismos neurológicos: 1 - Formação de placas senis a partir do peptídeo beta amiloide $(\mathrm{A} \beta)$, que ao se acumular nas regiões límbicas e corticais, promovem alterações funcionais nas sinapses e dendritos, além de inflamções decorrentes da ativação dos astrócitos e da micróglia, o que leva à morte de neurônios em consequência de danos celulares e bioquímicos, formação de radicais livres e espécies reativas de oxigênio (Umeda et al., 2011); 2 - Formação de emaranhados neuro fibrilares intracelulares em decorrência da fosforilação da proteína tau (Phospho-Tau), que prejudica o funcionamento estrutural e neuronal promovendo a atrofia cerebral, principalmente, da região temporal, hipocampo e do córtex entorrinal, o que compromete as funções cognitivas e os distúrbios comportamentais (Sanford, 2017). 
A DA é considerado um grave problema na saúde mundial, com elevado aumento da incidência em decorrência de vários fatores, dentre eles, o envelhecimento populacional, estilo de vida, assistência à saúde e, embora haja muitos estudos, não existe um tratamento eficaz para sua cura (ABRAz, 2019). Porém, tratamentos não farmacológicos têm apresentado bons resultados para amenizar as perdas cognitivas e motoras apresentadas por seus pacientes, como terapia cognitiva, terapia ocupacional, exercícios físicos e, mais recentemente tem ganho destaque nos estudos, a terapia por fotobiomodulação (Cardoso; Lopes-Martins; Gomes da Silva, 2020).

A fotobiomodulação (Photobiomodulation - PBM) é uma técnica de aplicação de luz vermelha ou infravermelha próxima, de baixa intensidade, de forma não invasiva, que estimula ou inibe células e tecidos através de mecanismos fotoquímicos (Hamblin, 2016a). Seus efeitos terapêuticos estão relacionados ao uso de lasers de baixa potência e diodos emissores de luz (LEDs), denominados de Terapia a Laser de baixa potência (Low Level Laser Therapy - LLLT), o qual já foi proposto no tratamento de distúrbios degenerativos, como Doença de Parkinson e Alzheimer, e de doenças psiquiátricas (Naeser; Hamblin, 2011), pois seus efeitos promovem respostas neuro protetoras, melhorias no fluxo sanguíneo, na neurogênese e no metabolismo, além da diminuição da inflamação e do estresse oxidativo (Freitas; Hamblin, 2016).

A PBM tem sido bastante discutida, porém muitas lacunas ainda são percebidas quanto aos métodos de aplicação, duração e efeitos reais no tratamento da Doença de Alzheimer, o que leva a relevância desse estudo, cujo objetivo é discutir, através de revisão integrativa, o impacto terapêutico da fotobiomodulação no tratamento da Doença de Alzheimer.

\section{Metodologia}

\section{Tipo de Estudo}

Trata-se de uma revisão narrativa da literatura, explorando dados qualitativos e quantitativos de estudos por meio de uma busca sistemática sobre o impacto do uso terapêutico da fotobiomodulação no tratamento da Doença de Alzheimer. O método de pesquisa utilizado objetiva traçar uma análise sobre o conhecimento construído em pesquisas anteriores sobre o tema, possibilitando a síntese de vários estudos já publicados, permitindo a geração de novos conhecimentos, pautados nos resultados apresentados pelas pesquisas anteriores. O presente estudo foi desenvolvido seguindo as seis etapas do método de uma revisão definida por Botelho et al. (2011) seguiu os princípios metodológicos de um estudo qualitativo de revisão, segundo as orientações definidas por Pereira et al. (2018).

\section{Identificação do tema e formulação da pergunta norteadora}

A questão de pesquisa foi formulada a partir da utilização da estratégia PICOS, que representa um acrônimo do inglês Population (paciente ou problema), Intervention (Intervenção), Comparison (Comparação), Outcomes (Desfecho) e Study design (Desenho do estudo), a partir da aplicação da estratégia, definiu-se como pergunta norteadora: "Qual o impacto do uso terapêutico da fotobiomodulação em pacientes com Doença de Alzheimer?".

A estratégia de busca incluiu os seguintes descritores: (Photobiomodulation, Alzheimer's Disease, Transcranial Photobiomodulation, Cognition) com seus respectivos termos correspondentes (MeSH terms) em Português e Espanhol. O operador booleano OR foi aplicado entre os termos. A busca foi realizada nas bases de dados PubMed, Medical Literature Analysis and Retrieval System Online (MEDLINE), Literatura Latino-Americana e do Caribe em Ciências da Saúde (LILACS) e Scientific Electronic Library Online (Scielo). Buscas manuais de estudo também foram realizadas e consideradas: (1) lista de referência de artigos incluídos durante o processo de busca; (2) Base de registros de Estudos Randomizados.

\section{Estabelecimento de critérios de inclusão e exclusão}

Definiu-se como critérios de inclusão: estudos publicados entre janeiro de 2011 a janeiro de 2021 em Inglês, 
Português ou Espanhol, que descrevessem características da fotobiomodulação no tratamento da DA. Foram definidos como elegíveis todos os tipos de estudos, a saber - relatos ou séries de casos, estudos retrospectivos, prospectivos, ensaios clínicos randomizados, revisões da literatura, protocolos e guidelines. Foram excluídos estudos realizados em animais, pesquisas in vitro.

\section{Identificação dos estudos pré-selecionados e selecionados}

A seleção dos estudos foi realizada por dois revisores cegos e independentes. Na primeira etapa, foram lidos os títulos e resumos dos estudos encontrados a partir da busca descrita no item anterior, sendo aplicado os critérios de elegibilidade previamente definidos nesta revisão. Os estudos selecionados na primeira etapa, foram lidos integralmente, sendo novamente aplicado os critérios de elegibilidade, determinando, então, a inclusão ou não na pesquisa.

Após avaliar e obter consenso sobre os estudos incluídos, os dados foram extraídos e apresentados de forma descritiva.

\section{Análise e interpretação dos resultados}

Após a cuidadosa análise e interpretação dos resultados, os autores organizaram a apresentação desta revisão em uma tabela contendo os dados referentes ao método empreendidos por cada autor, seguido dos resultados encontrados. Além disso, os dados encontrados foram contrapostos com o que já havia disponível na literatura sobre o tema na sessão de discussão.

\section{Resultados e Discussão}

\section{Seleção dos Artigos}

No total foram identificados 154 artigos referentes aos descritores selecionados, onde 126 foram descartados após estudos com animais, textos não disponíveis na íntegra e publicações duplicadas. Foram selecionados para leitura 28 artigos, dos quais 21 não respondiam ao objetivo do estudo ou não apresentaram procedimentos descritos de forma clara. 7 artigos responderam a todos os critérios e objetivo do presente estudo, os detalhes de cada um deles são apresentados na Tabela 1.

\section{Análise dos Resultados}

As pesquisas que envolvem a PBM em pacientes com Doença de Alzheimer são ainda escassas e relativamente recentes, porém, os resultados já obtidos são significativos e promissores, pois apresentam indícios que é possível reverter alguns quadros de perdas cognitivas através de alterações celulares e melhorias circulatórias, medidas através de testes cognitivos e exames de imagem, respectivamente (Lim, 2018). 
Tabela 1. Artigos que correspondem aos critérios de elegibilidade e inclusão do estudo

\begin{tabular}{|c|c|}
\hline Autor (ano) & Métodos \\
\hline \multirow[t]{5}{*}{ Maksimovich (2015) } & $\begin{array}{l}89 \text { indivíduos (separados por grupos de estágios de DA e } \\
\text { controle) }\end{array}$ \\
\hline & $\begin{array}{l}\text { Testes cognitivos: MEEM e Avaliação Clínica de } \\
\text { Demência. }\end{array}$ \\
\hline & Testes de imagem: Cintilografia, Reoencefalografia, TC e \\
\hline & RM. \\
\hline & $\begin{array}{l}\text { PBM: Transcateter com laser de luz vermelha de baixa } \\
\text { energia }(20 \mathrm{~nm}) \text {, através de um cateter na artéria femoral. }\end{array}$ \\
\hline \multirow[t]{4}{*}{ Berman et al. (2017) } & 11 indivíduo (experimental e controle) com DA. \\
\hline & Testes cognitivos: MEEM, ADAS-Cog. \\
\hline & Teste de imagem: EEG \\
\hline & $\begin{array}{l}\text { PBM: Transcranial, } 6 \text { min diário, por } 28 \text { dias consecutivos } \\
(1060-1080 \mathrm{~nm}, 10 \mathrm{~Hz}, 50 \%)\end{array}$ \\
\hline \multirow{4}{*}{$\begin{array}{l}\text { Zomorrodi et al. } \\
(2017)\end{array}$} & 1 indivíduo com DA. \\
\hline & Testes cognitivos: MEEM, ADAS-Cog, ADCS-ADL \\
\hline & Teste de imagem: EEG. \\
\hline & $\begin{array}{l}\text { PBM: transcraniano e intranasal, } 1 \text { vez por noite, } 6 \text { noites } \\
\text { por semana, durante } 17 \text { semanas }(810 \mathrm{~nm}, 40 \mathrm{~Hz}, 50 \%)\end{array}$ \\
\hline \multirow{4}{*}{$\begin{array}{l}\text { Saltmarche et al. } \\
(2017)\end{array}$} & 5 indivíduos com DA. \\
\hline & Testes cognitivos: MMSE e ADAS-Cog. \\
\hline & PBM: intranasal, $25 \mathrm{~min}, 2$ vezes por semana, $(810 \mathrm{~nm}, 10$ \\
\hline & $\begin{array}{l}\mathrm{Hz}, 50 \%) \text {, e também o Transcraniano }(810 \mathrm{~nm}, 10 \mathrm{~Hz}, 50 \%) \\
\text { onde sua aplicação foi dividida em: } 2 \text { vezes por semana } \\
\text { durante as visitas na clínica durante as primeiras } 2 \text { semanas, } \\
\text { então aplicado apenas } 1 \text { vez por semana, para cada um, nas } 10 \\
\text { semanas seguintes. }\end{array}$ \\
\hline
\end{tabular}

Salehpour et al. 1 indivíduo com DA.

(2019) Testes Cognitivos: MoCA, WMQ, AST e PSM.

PBM: Transcraniano (LEDs vermelhos $635 \mathrm{~nm}$ ) e almofada corporal (NIR $810 \mathrm{~nm})$ e Intranasal $(810 \mathrm{~nm}, 10 \mathrm{~Hz})$, por $25 \mathrm{~min}, 2$ vezes ao dia, durante 4 semanas.

Chao (2019)

Horner, Berger, Gibas (2020)

Nagy et al. (2021)

Testes cognitivos: MEEM, ADAS-Cog e NPI
8 participantes com DA (controle e experimental)

Testes de imagem: Siemens 3 Tesla trio scanner, RM.

PBM: transcranial e intranasal, por 20min, e vezes por semana, durante 12 semanas. $(810 \mathrm{~nm}, 40 \mathrm{~Hz}, 50 \%)$

1 indivíduo com DA leve.

Testes cognitivos: MoCA, Desenho do relógio, Série de 7 subtrações e Fluência de 1 min.

PBM: transcranial e intranasal, 3 vezes por semana, por 20 $\min .(810 \mathrm{~nm}, 40 \mathrm{~Hz}, 50 \%)$

Associada a dieta cetogênica

60 indivíduos (experimental e controle) com DA.

Testes Cognitivos: MoCA-B Básico.

PBM: Dispositivo intranasal e relógio a laser na arterial radial, $3 \mathrm{~min}, 2$ vezes por dia, 3 vezes na semana, durante 3 meses $(650 \mathrm{~nm})$.

Associada a exercício físico aeróbico
Tendência de melhora do grupo experimental, após PBM, no funcionamento executivo, desenho do relógio, recordação imediata, memória de práxis, atenção visual, troca de tarefas, além da amplitude e conectividade de ECG.

A PBM promoveu melhoras cognitivas apresentadas em todos os testes cognitivos (MMSE, ADAS-Cog, ADCS-ADL).

A PBM transcraniana + intranasal mostrou melhora significativa na cognição, habilidades funcionais para atividades da vida diária e melhor qualidade de vida.

A PBM multimodal, em uso doméstico apresenta melhorias nas funções cognitivas observadas através de testes cognitivos (MoCA, WMQ, AST e PSM).

Não apresentou diferença significativa entre os grupo do ADAS-Cog, mas uma tendência para pontuação mais baixa no NPI para o grupo controle.

Melhora significativa da cognição após PBM: 20/30 pontos no MoCA inicial, para $26 / 30$ e teste de fluência de $1 \mathrm{~min}$ melhorou de 19 para 33, após PBM.

Irradiação a laser intranasal combinada com acupuntura de relógio a laser e exercício aeróbico promove melhorias cognitivas demonstradas através do teste de MoCA-B básico. 
Assessment Scale-Cognitive; ADCS-ADL - Alzheimer's Disease Cooperative Study Activities of Daily Living Inventory; MoCA - Montreal Cognitive Assessment; WMQ - WM Questionnaire; AST - Alberta Smell Test; PSM - Physical Self Maintenance; NPI= Neuropsychiatric Inventary; LED - Light Emitting Diode; NIR - Near Infrared Radiation. Fonte: Autoria Própria

\section{Discussão}

Dentre as causas da DA, ainda não exploradas totalmente, sabe-se que o seu desenvolvimento está normalmente conexo a Apolipoproteína E4 (apoE4), o acúmulo de $\beta$-amiloide no tecido cerebral, ao alelo tau e a disfunções vasculares cerebrais (Sakay et al., 2014). A relação entre DA e distúrbios vasculares cerebrais tornou-se importante na patogênese e etiologia da doença, pois o cérebro é considerado um órgão com maior perfusão no corpo humano e, com isso, qualquer alteração no fluxo sanguíneo pode gerar hipóxia, distúrbios no metabolismo e isquemia tecidual (Maksimovich, 2015). Com isso, alguns estudos foram realizados buscando novos métodos de tratamento não farmacológicos para distúrbios cognitivos, através da restauração do fluxo sanguíneo nas artérias e capilares distais, dentre eles o transcateter a laser (Barrett, GonzalezLima, 2013).

Os efeitos positivos nas respostas cognitivas e no controle da demência, apresentados após PBM por transcateter com laser de luz vermelha de baixa energia $(20 \mathrm{~nm})$, através de um cateter na artéria femoral, está relacionado ao estímulo da angiogênese, a qual promove revascularização colateral e dos capilares, o que, consequentemente, promove melhorias na nutrição cerebral e redução de hipóxia. Estas por sua vez, melhoram a retirada de $\beta$-amiloide e restauração do tecido cerebral, e ainda, promovem a neurogênese, através da elevação dos recursos enérgicos causados às mitocôndrias (Maksimovich, 2015). Outra técnica de PBM estudada como meio terapêutico às doenças neurodegenerativas, é a aplicação de energia luminosa ao cérebro, a PBM transcraniana (tPBM), onde seu efeito está relacionado a melhoria do fluxo sanguíneo no cérebro e, consequentemente, da distribuição e consumo de oxigênio, além da maior produção de adenosina trifosfato (ATP) e maior atividade das mitocôndrias (Hamblin, 2016b).

O mecanismo da tPBM com radiação de luz infravermelha próxima (NIR) está baseado na capacidade da luz em promover a foto-oxidação do citocromo oxidase $(\mathrm{CCO})$, o qual é responsável pela catalização do metabolismo do oxigênio e consequente produção de ATP (Freitas e Hamblin, 2016). A foto-oxidação do CCO ativa o uso de oxigênio nas mitocôndrias e a produção de ATP intracelular, com isso, a grande concentração de mitocôndrias presentes nas células e neurônios, irão ativar o processo metabólico do oxigênio mitocondrial, gerando assim, maior atividade funcional e aumento das ligações sinápticas, melhorando assim, as respostas cognitivas (Gonzalez-Lima, Barksdale, Rojas, 2014).

A tPBM visa o estímulo de uma área do cérebro específica, de acordo com a patologia ou distúrbio tratado, através da aplicação de um feixe de luz, ou vários feixes, em determinada área da cabeça. O feixe de luz pode ter origem de um laser ou de diodo emissor de luz (LED) de forma pulsada ou contínua (Hennessy, Hamblin, 2017). Dentre outras vantagens como, não ser invasivo e não apresentar efeitos colaterais, a tPBM ainda pode ser aplicada paralelo a outros tratamentos (Johnstone et al., 2016), como nos estudos de Horner, Berger e Gibas (2020) e Nagy et al. (2021), os quais associaram-na a dieta cetogênica e exercício aeróbico, respectivamente.

Outro dos métodos aplicados nos estudos apresentados é a terapia PBM intranasal (i-PBM), a qual surgiu como uma forma de suplantar alguns limites da tPBM em atingir determinadas áreas do cérebro, como córtex pré-frontal e áreas límbicas (Cassano et al., 2019). A i-PBM é ministrada pelas narinas ou via cavidades nasais, através de uma ou duas sondas pequenas composta por laser/LED, cujos efeitos estão relacionados a melhora das disfunções neuronais, como por exemplo, a DA (Saltmarche, et al., 2017; Chao, 2019).

Os resultados obtidos pela i-PBM, cuja aplicação ocorre através das narinas, utilizados nos estudos de Zomorrodi et al. (2017), Saltmarche et al. (2017), Salehpour et al. (2019), Horner, Berger, Gibas (2020) e Nagy et al. (2021), apresentam um 
efeito sistêmico em decorrência da irradiação de células e componentes sanguíneos, que leva a melhoria da oxigenação e elevação dos níveis de ATP no cérebro (Hamblin, 2016b, Hennessy, Hamblin, 2016). Além desses efeitos, a i-PBM pode ainda liberar óxido nítrico (NO) e modular a formação de espécie reativas de oxigênio (ERO), o que explica sua ação neuro protetora (Salehpour et al., 2020) e resultados positivos das funções executivas e atividades da vida diária.

Todos os métodos de PBM aplicados nos estudos apresentam resultados importantes no controle das perdas das funções cognitivas, porém não se pode afirmar qual a melhor dosagem quanto ao comprimento de onda, densidade de potência e duração da exposição, embora os estudos aqui apresentados usarem especificações bastante próximas. O que a literatura sugere é que o comprimento de onda do laser de baixa potência u LEDs esteja dentro da janela terapêutica entre vermelho e infravermelho próximo $(660 \mathrm{~nm}$ a $1.100 \mathrm{~nm})$, com fluência até $60 \mathrm{~J} / \mathrm{cm}^{2}$ para que os resultados sejam mais significantes (Enengla, Hamblin, Dungel, 2020).

Os efeitos da PBM, embora ainda necessite de vários estudos que elucidem alguns pontos em sua aplicabilidade, apresenta resultados já perceptíveis quanto as mudanças benéficas que promove ao cérebro: alterações metabólicas (ATP), aumento do fluxo sanguíneo, neuro proteção, controle da produção de ERO e NO, efeito anti-inflamatório, neurogênese e sinaptogênese (Hamblin, 2019).

Os estudos em humanos ainda são poucos, mas o que se percebe é que a PBM promove respostas importantes as pessoas com DA, tais como, melhoria cognitiva (raciocínio, fluência, atenção), melhor desempenho nas atividades da vida diária e aumento da qualidade de vida (Saltmarche et al., 2017, Zomorrodi et al., 2017).

\section{Conclusão}

A Fotobiomodulação, embora ainda apresente algumas divergências quanto a sua aplicabilidade, tem um impacto positivo no tratamento da Doença de Alzheimer, pois através de inúmeras alterações que promove no cérebro, possibilita a melhora das funções cognitivas, habilidades funcionais diárias e qualidade de vida. Esses resultados são decorrentes da melhoria do fluxo sanguíneo, neurogênese, sinaptogênese, dentre outros, os quais reestabelecem estruturas danificadas do cérebro que contribuem para o surgimento e agravamento da doença.

Mais estudos são necessários para elucidar as lacunas ainda existentes sobre a Fotobiomodulação em humanos com Doença de Alzheimer, o que irá contribuir para sua prevenção, tratamento e controle dessa doença que avança em todo o mundo.

\section{Referências}

ABRAz, Associação Brasileira de Alzheimer. (2019). Falar sobre a Doença de Alzheimer (DA) é uma questão de saúde pública. http://abraz.org.br/web/2019/08/05/falar-sobre-a-doenca-de-alzheimer-da-e-uma-questao-de-saude-publica/.

ABRAz, Associação Brasileira de Alzheimer. (2021). O que é Alzheimer. https://abraz.org.br/2020/sobre-alzheimer/o-que-e-alzheimer-2/ .

Barrett, D. W., \& Gonzalez-Lima, F. (2013) Transcranial Infrared Laser Stimulation Produces Beneficial Cognitive and Emotional Effects in Humans. Neuroscience, 29, 13-23. 10.1016/j.neuroscience.2012.11.016.

Berman, M. H., Halper, J. P., Nichols, T. W., Jarret, H. Lundy, A., \& Huang, J. H. (2017). Photobiomodulation with Near Infrared Light Helmet in a Pilot, Placebo Controlled Clinical Trial in Dementia Patients Testing Memory and Cognition. J Neurol Neurosci. 8(1): 10.21767/2171-6625.1000176.

Botelho, L. L. R., Cunha, C. C. de A., \& Macedo, M. (2011). O método da revisão integrativa nos estudos organizacionais. Gestão e Sociedade, 5(11), 121. https://doi.org/10.21171/ges.v5i11.1220

Cardoso, F. D. S., Lopes-Martins, R. A. B, \& Gomes da Silva, S. (2020). Therapeutic potential of photobiomodulation In Alzheimer's disease: a systematic review. J Lasers Med Sci. 11(suppl 1): S16-S22. 10.34172/jlms.2020.S3.

Cassano, P., Tran, A. P., Katnani, H., Bleier, B. S., Hamblin, M. R., Yuan, Y., \& Fangi, Q. (2019). Selective photobiomodulation for emotion regulation: model-based dosimetry study. Neurophotonics. Jan; 6(1): 015004. 10.1117/1.NPh.6.1.015004.

Cavalcanti, J. L. S., \& Engelhardt, E. (2012). Aspectos da Fisiopatologia da Doença de Alzheimer Esporádica. Rev Bras de Neurologia. 48(4): 21-29. 
Chao, L. L. (2019). Effects of Home Photobiomodulation Treatments on Cognitive and Behavioral Function, Cerebral Perfusion, and Resting-State Functional Connectivity in Patients with Dementia: A Pilot Trial. Photobiomodulation, Photomedicine, and Laser Surgery. 37(3). doi.org/10.1089/photob.2018.4555.

Enengla, J., Hamblin, M. R., \& Dungele, P. (2020). Photobiomodulation for Alzheimer's Disease: Translating Basic Research to Clinical Application. Journal of Alzheimer's Disease. 75:1073-1082. 10.3233/JAD-191210.

Freitas, L. F.; \& Hamblin, M. R. (2016). Proposed Mechanisms of Photobiomodulation or Low-Level Light Therapy. IEEE J Sel Top Quantum Electron. (3): 7000417. 10.1109/JSTQE.2016.2561201.

Gonzalez-Lima, F., Barksdale, B. R., \& Rojas, J. C. (2014). Mitochondrial respiration as a target for neuroprotection and cognitive enhancement. Biochem Pharmacol. 88(4):584-93. 10.1016/j.bcp.2013.11.010.

Hamblin, M. R. (2016a) Photobiomodulation or low-level laser therapy. J Biophotonics. 9(11-12): 1122-1124. 10.1002/jbio.201670113.

Hamblin, M. R. (2016b). Shining light on the head: Photobiomodulation for brain disorders. BBA Clin 6 113-124, 10.1016/j. bbacli.2016.09.002.

Hamblin, M. R., (2019). Photobiomodulation for Alzheimer's Disease: Has the Light Dawned? Photonics. 6(3): 77. 10.3390/photonics6030077.

Hennessy M., \& Hamblin, M. R. (2017). Photobiomodulation and the brain: a new paradigm. J. Opt. 19. 013003. 10.1088 / 2040-8986 / 19/1/013003

Horner S., Berger L., Gibas K. (2020). Nutritional Ketosis and photobiomodulation remediate mitochondria warding off Alzheimer's disease in a diabetic, ApoE4+ patient with mild cognitive impairment: A case report. Photodiagnosis and Photodynamic Therapy. 30:101777. doi.org/10.1016/j.pdpdt.2020.101777.

Johnstone, D. M., Moro, C., Stone, J., Benabid, A-L., \& Mitrofanis, J. (2016) Turning On Lights to Stop Neurodegeneration: The Potential of Near Infrared Light Therapy in Alzheimer's and Parkinson's Disease. Front. Neurosci. 9:500. 10.3389/fnins.2015.00500

Lim, L. (2018). The Growing Evidence for Photobiomodulation as a Promising Treatment for Alzheimer's Disease. Journal of Biosciences and Medicines, 2018, 6, 100-110. 10.4236/jbm.2018.612010.

Maksimovich, I. V. (2015). Dementia and Cognitive Impairment Reduction after Laser Transcatheter Treatment of Alzheimer's Disease. World Journal of Neuroscience, 5, 189-203. 10.4236/wjns.2015.53021.

Martinelli, M. (2020). A Importância da Identificação Precoce da Doença de Alzheimer. Disponível em https://idosos.com.br/identificacao-precoce-da-doencade-alzheimer/. Acesso em Abril de 2021.

Nagy, E. N., Ali, A. Y., Behiry, M. E., Naguib, M. M., \& Elsayed, M. M. (2021). Impact of Combined Photo-Biomodulation and Aerobic Exercise on Cognitive Function and Quality-of-Life in Elderly Alzheimer Patients with Anemia: A Randomized Clinical Trial. Int J Gen Med. 14: 141-152. 10.2147/IJGM.S280559.

Pereira, A. S., Shitsuka, D. M., Parreira, F. J., \& Shitsuka, R. (2018). Método Qualitativo, Quantitativo ou Quali-Quanti. In Metodologia da Pesquisa Científica. https://repositorio.ufsm.br/bitstream/handle/1/15824/Lic_Computacao_Metodologia-Pesquisa-Cientifica.pdf?sequence=1. Acesso em: 28 março 2020 .

Sakai, K., Boche, D., Carare, R., Johnston, D., Holmes, C., Love, S. \& Nicoll, J. A. R. (2014) A Immunotherapy for Alzheimer's Disease: Effects on apoE and Cerebral Vasculopathy. Acta Neuropathologica, 128, 777-789. 10.1007/s00401-014-1340-9.

Salehpour, F., Gholipour-Khalili, S., Farajdokht, F., Kamari, F., Walski, T., Hamblin, M. R., DiDuro, J. O., \& Cassanoa, P. (2020). Therapeutic potential of intranasal photobiomodulation therapy for neurological and neuropsychiatric disorders: a narrative review. Rev Neurosci. 28; 31(3): 269-286. 10.1515/revneuro-2019-0063.

Salehpour, F., Hamblin, M. R., \& DiDuro, J. O. (2019). Rapid Reversal of Cognitive Decline, Olfactory Dysfunction, and Quality of Life Using MultiModality Photobiomodulation Therapy: Case Report. Photobiomodulation, Photomedicine, and Laser Surgery. 37 (3): 159-167. 10.1089/photob.2018.4569.

Saltmarche, A. E., Naeser, M. A., Ho, K. F., Hamblin, M. R., \& Lim, L. (2017). Significant Improvement in Cognition in Mild to Moderately Severe Dementia Cases Treated with Transcranial Plus Intranasal Photobiomodulation: Case Series Report. Photomedicine and Laser Surgery. 35(8): 432-441. 10.1089/pho.2016.4227.

Sanford, A. M. (2017). Mild cognitive impairment. Clin Geriatr Med. 33(3):325-337. 10.1016/j.cger.2017.02.005.

Zomorrodi, R., Saltmarche, A. E., Loheswaran, G., Ho, K. F., \& Lim, L. (2017). Complementary EEG Evidence for a Significantly Improved Alzheimer's Disease Case after Photobiomodulation Treatment. Presented at: Alzheimer's Association International Conference, July 14-20. 